[6] D. P. Bertsekas, "Dynamic programming and suboptimal control: A survey from ADP to MPC," Fundam. Issues Control, Eur. J. Control, vol. 11, no. 4-5, 2005.

[7] D. P. Bertsekas, Separable dynamic programming and approximate decomposition methods Mass. Inst. Technol., Cambridge, MA, Rep. LIDS 2684, 2006.

[8] D. P. Bertsekas, Dynamic Programming and Optimal Control, 3rd ed. Belmont, MA: Athena Scientific, 2007, vol. II.

[9] F. Blanchini, "Set invariance in control-A survey," Automatica, vol. 35, pp. 1747-1768, 1999.

[10] M. Caramanis and A. Sharifnia, "Near optimal manufacturing flow controller design," Int. J. Flexible Manuf. Syst., vol. 3, pp. 321-336, 1991.

[11] J. R. Deller, "Set membership identification in digital signal processing," IEEE ASSP Mag., pp. 4-20, Oct. 1989.

[12] S. D. Dreyfus, Dynamic Programming and the Calculus of Variations. New York: Academic, 1965.

[13] A. M. Geoffrion, "Elements of large-scale mathematical programming, I, II," Manage. Sci., vol. 16, pp. 652-675, 1970, 676-691.

[14] A. M. Geoffrion, "Lagrangian relaxation for integer programming," Math. Program. Stud., vol. 2, pp. 82-114, 1974.

[15] S. B. Gershwin, Manufacturing Systems Engineering. Englewood Cliffs, NJ: Prentice-Hall, 1993.

[16] J. Kimemia, S. B. Gershwin, and D. P. Bertsekas, "Computation of production control policies by a dynamic programming technique," in Analysis and Optimization of Systems, A. Bensoussan and J. L. Lions, Eds. New York: Springer-Verlag, 1982, pp. 243-269.

[17] R. L. Kosut, M. K. Lau, and S. P. Boyd, "Set-membership identification of systems with parametric and nonparametric uncertainty," IEEE Trans. Autom. Control, vol. 37, no. 7, pp. 929-941, Jul. 1992.

[18] J. Kimemia, "Hierarchical control of production in flexible manufacturing systems," Ph.D. dissertation, Dept. Elect. Eng. Comput. Sci., Mass. Inst. Technol., Cambridge, MA, 1982.

[19] L. S. Lasdon, Optimization Theory for Large Systems. New York: Macmillian, 1970.

[20] D. Q. Mayne, "Control of constrained dynamic systems," Eur. J. Control, vol. 7, pp. 87-99, 2001.

\section{On the Rendezvous Problem for Multiple Nonholonomic Agents}

Dimos V. Dimarogonas and Kostas J. Kyriakopoulos

Abstract-In this note, a decentralized feedback control strategy that drives a system of multiple nonholonomic unicycles to a rendezvous point in terms of both position and orientation is introduced. The proposed nonholonomic control law is discontinuous and time-invariant and using tools from nonsmooth Lyapunov theory and graph theory the stability of the overall system is examined. Similarly to the linear case, the convergence of the multi-agent system relies on the connectivity of the communication graph that represents the inter-agent communication topology. The control law is first defined in order to guarantee connectivity maintenance for an initially connected communication graph. Moreover, the cases of static and dynamic communication topologies are treated as corollaries of the proposed framework.

Index Terms-Cooperative control, decentralized control, nonholonomic agents.

\section{INTRODUCTION}

In this note, the problem of rendezvous convergence for a system of multiple nonholonomic unicycles in terms of both position and orientation is considered. The rendezvous problem has been extensively approached recently, addressing the control design issue from several perspectives. Recent results include [6], [11], [12], [14], [17], and [19]. In most cases, linear models of motion are taken into account, while the information exchange topology is considered both static and dynamic, as well as bidirectional or unidirectional. A recent review of the various approaches of the rendezvous problem for linear models of motion is [21].

In this note, a decentralized control strategy that drives a system of multiple kinematic unicycles to rendezvous is presented. The proposed nonholonomic feedback law is discontinuous and time invariant, something expected, as nonholonomic systems do not satisfy Brocketts necessary smooth feedback stabilization condition [3]. These controllers have in general better convergence properties than time-varying ones. An experimental comparison between these two types of controllers that supports our preference to time-invariant strategies has appeared in [13], where the authors concluded that time-varying controllers were too slow and oscillatory for most practical cases. In contrast, time-invariant controllers achieved a significantly better performance.

A first contribution of this note is that the control law is first defined in order to guarantee connectivity maintenance. Hence, if the communication graph is initially connected, it remains connected throughout the closed loop system evolution and rendezvous is reached, under the proposed control law. Connectivity preserving for linear agents was

Manuscript received November 22, 2005; revised May 9, 2006 and November 22, 2006. Recommended by Asssociate Editor F. Bullo. This work was supported by the European Commission through contract I-SWARM (IST-2004507006), http://www.i-swarm.org/. Preliminary versions of this work have appeared in [7] and [8].

The authors are with the Control Systems Laboratory, Mechanical Engineering Department, the National Technical University of Athens, Athens 15780, Greece (e-mail: ddimar@mail.ntua.gr; kkyria@mail.ntua.gr).

Color versions of one or more of the figures in this paper are available online at http://ieeexplore.ieee.org.

Digital Object Identifier 10.1109/TAC.2007.895897 
considered in [1] and [12]. The weaker cases of static and dynamic interconnection topology are treated as corollaries of the proposed framework. Our treatment is similar to that of [15], where merely static interconnection topology is considered. Furthermore, the authors of [15] use a similar control strategy to that of [24], which is time varying periodic and smooth. Hence, it provides in general worse convergence results with respect to the time invariant case encountered in this note. A key drawback of the current approach is the need of knowledge of a global coordinate frame with respect to the orientation of each agent, as opposed to the angular velocity open-loop control law of [15]. In contrast, only relative knowledge of the neighboring agents positions is needed.

The rest of the note is organized as follows. Section II describes the system and the problem treated in this note. Assumptions regarding the communication topology between the agents are presented and modelled in terms of an undirected graph. Section III begins with some background on graph and matrix theory and nonsmooth analysis that is used in the sequel and proceeds with the introduction of the decentralized nonsmooth time invariant feedback strategy that drives the multiagent team to a common point in the state space as well as the corresponding stability analysis. We first treat the case of connectivity maintenance while the cases of static and dynamic communication topology are treated next. Computer simulations are included in Section IV while Section V summarizes the results of this note.

\section{SySTEM AND PROBLEM DEFINITION}

Consider a system of $N$ nonholonomic point agents operating in the same workspace $W \subset \mathbb{R}^{2}$. Let $q_{i}=\left[x_{i}, y_{i}\right]^{T} \in \mathbb{R}^{2}$ denote the position of agent $i$. The configuration space is formed by vectors of the form $q=$ $\left[q_{1}, \ldots, q_{N}\right]^{T}$. Each of the $N$ mobile agents has a specific orientation $\theta_{i}$ with respect to the global coordinate frame. The orientation vector of the agents is represented by $\theta=\left[\theta_{1}, \ldots, \theta_{N}\right]^{T}$. The configuration of each agent is represented by $p_{i}=\left[q_{i}, \theta_{i}\right]^{T} \in \mathbb{R}^{2} \times(-\pi, \pi]$. Agent motion is described by the following nonholonomic kinematics

$$
\begin{aligned}
& \dot{x}_{i}=u_{i} \cos \theta_{i} \\
& \dot{y}_{i}=u_{i} \sin \theta_{i}, \quad i \in \mathcal{N}=[1, \ldots, N] \\
& \dot{\theta}_{i}=\omega_{i}
\end{aligned}
$$

where $u_{i}, \omega_{i}$ denote the translational and rotational velocity of agent $i$, respectively.

The design objective is to construct feedback controllers that lead the multiagent system to rendezvous, i.e. all agents should converge to a common point $p^{*}=\left[q^{*}, \theta^{*}\right]^{T}$ in the state space. Each agent is assigned to a specific subset $N_{i}$ of the rest of the team, called agent $i$ 's communication set, that includes the agents with which it can communicate in order to achieve the desired goal. Interagent communication is encoded in terms of a communication graph [18]:

Definition 1: The communication graph $\mathcal{G}=\{V, E\}$ is an undirected graph that consists of a set of vertices $V=\{1, \ldots, N\}$ indexed by the team members and a set of edges, $E=\{(i, j) \in V \times V \mid i \in$ $\left.N_{j}\right\}$ containing pairs of nodes that represent interagent communication specifications.

Each agent has only knowledge of the state of agents that belong to its communication set at each time instant. This fact highlights the decentralized nature of the approach. We also assume that the communication graph is undirected, in the sense that $i \in N_{j} \Leftrightarrow j \in N_{i}, \forall i$, $j \in \mathcal{N}, i \neq j$. It is obvious that $(i, j) \in E$ iff $i \in N_{j} \Leftrightarrow j \in N_{i}$. The control design will have the form

$$
\begin{aligned}
& u_{i}=u_{i}\left(p_{i}, p_{j}\right) \\
& \omega_{i}=\omega_{i}\left(p_{i}, p_{j}\right)
\end{aligned}, j \in N_{i}, i \in \mathcal{N}
$$

according to the limited communication capabilities of each agent. Hence the control law of each agent depends on its own configuration $p_{i}$ as well as the configurations $p_{j}$ of agents belonging to its communication set.

The problems treated in this note are summarized as follows: we derive a set of control laws of the form (2) that drives the agents to a rendezvous point and, at the same time, guarantees that if the initially formed communication graph is connected, then it remains connected throughout the system evolution. Furthermore, the cases of static and dynamic communication topology without connectivity maintenance are treated as corollaries of the proposed framework.

\section{CONTROL StRATEGY AND Stability ANALYSIS}

In this section, the proposed feedback law and the corresponding stability analysis of the system are presented. The mathematical tools required are discussed in the next two subsections.

\section{A. Tools From Algebraic Graph Theory and Matrix Analysis}

In this subsection, we review some tools from graph theory and matrix analysis that we shall use in the stability analysis of the proposed control framework. The following analysis on graph theory can be found in [2], while the elements from matrix analysis in [10], [16].

For an undirected graph $\mathcal{G}$ with $n$ vertices the adjacency matrix $A=A(\mathcal{G})=\left(a_{i j}\right)$ is the $n \times n$ matrix given by $a_{i j}=1$, if $(i, j) \in E$ and $a_{i j}=0$, otherwise. If there is an edge connecting two vertices $i, j$, i.e., $(i, j) \in E$, then $i, j$ are called adjacent. A path of length $r$ from a vertex $i$ to a vertex $j$ is a sequence of $r+1$ distinct vertices starting with $i$ and ending with $j$ such that consecutive vertices are adjacent. If there is a path between any two vertices of the graph $\mathcal{G}$, then $\mathcal{G}$ is called connected (otherwise it is called disconnected). The degree $d_{i}$ of vertex $i$ is defined as the number of its neighboring vertices, i.e. $d_{i}=\{\# j:(i, j) \in E\}$. Let $\Delta$ be the $n \times n$ diagonal matrix of $d_{i}$ 's. The (combinatorial) Laplacian of $\mathcal{G}$ is the symmetric positive semidefinite matrix $\mathcal{L}=\Delta-A$. The Laplacian captures many interesting topological properties of the graph. Of particular interest in our case is the fact that for a connected graph, the Laplacian has a single zero eigenvalue and the corresponding eigenvector is the vector of ones, $\overrightarrow{\mathbf{1}}$.

The undirected graph $\mathcal{G}=(V, E)$ corresponding to a real symmetric $n \times n$ matrix $M$ is a graph with $n$ vertices indexed by $1, \ldots, n$ such that there is an edge between vertices $i, j \in V$ if and only if $M_{i j} \neq$ 0 , i.e., $(i, j) \in E \Leftrightarrow M_{i j} \neq 0$. A $n \times n$ real symmetric matrix with nonpositive off-diagonal elements and zero row sums is called a symmetric Metzler matrix. It is shown in [16] that all the eigenvalues of a symmetric Metzler matrix are non-negative and zero is a trivial eigenvalue. The multiplicity of zero as an eigenvalue of a symmetric Metzler matrix is one (i.e., it is a simple eigenvalue) if and only if the corresponding undirected graph is connected. The trivial corresponding eigenvector is the vector of ones, $\overrightarrow{\mathbf{1}}$. This result has been used in the proof of the consensus algorithm for single integrator kinematic agents presented in [17]. Its usefulness in the present framework is verified in the sequel.

\section{B. Tools From Nonsmooth Analysis}

We now review some elements from nonsmooth analysis that we use in the next sections. For a differential equation with a discontinuous right-hand side, we have the following definition.

Definition 2: [9] In the case of a finite dimensional state-space, the vector function $x($.$) is called a Filippov solution of \dot{x}=f(x)$, where $f$ is measurable and essentially locally bounded, if it is absolutely continuous and $\dot{x} \in K[f](x)$ almost everywhere where $K[f](x) \equiv$ $\overline{c o}\left\{\lim _{x_{i} \rightarrow x} f\left(x_{i}\right) \mid x_{i} \notin N_{0}\right\}$ and $N_{0}$ is a set of measure zero that contains the set of points where $f$ is not differentiable. 
Lyapunov theorems have been extended to nonsmooth systems in [4] and [22]. The following chain rule provides a calculus for the time derivative of the energy function in the nonsmooth case.

Theorem 1: [22] Let $x$ be a Filippov solution to $\dot{x}=f(x)$ on an interval containing $t$ and $V: \mathbb{R}^{n} \rightarrow \mathbb{R}$ be a Lipschitz and regular function. Then, $V(x(t))$ is absolutely continuous, $(d / d t) V(x(t))$ exists almost everywhere and

$$
\frac{d}{d t} V(x(t)) \epsilon^{a \cdot e \cdot \dot{\tilde{V}}(x):=} \bigcap_{\xi \in \partial V(x(t))} \xi^{T} K[f](x(t))
$$

where "a.e." stands for "almost everywhere".

In this theorem, $\partial V$ is Clarke's generalized gradient. The definition of the generalized gradient and of the regularity of a function can be found in [5]. In this note, the candidate Lyapunov function $V$ we use is smooth and hence regular, while its generalized gradient is a singleton which is equal to its usual gradient everywhere in the state space: $\partial V(x)=\{\nabla V(x)\} \forall x$.

We use the following nonsmooth version of LaSalle's invariance principle in the sequel:

Theorem 2: [22] Let $\Omega$ be a compact set such that every Filippov solution to the autonomous system $\dot{x}=f(x), x(0)=x\left(t_{0}\right)$ starting in $\Omega$ is unique and remains in $\Omega$ for all $t \geq t_{0}$. Let $V: \Omega \rightarrow \mathbb{R}$ be a time independent regular function such that $v \leq 0, \forall v \in \dot{\widetilde{V}}$ (if $\dot{\widetilde{V}}$ is the empty set then this is trivially satisfied). Define $S=\{x \in \Omega \mid 0 \in \dot{\tilde{V}}\}$. Then every trajectory in $\Omega$ converges to the largest invariant set, $M$, in the closure of $S$.

Uniqueness of solutions is guaranteed by the above definition of Filippov solutions, along with the measurability assumption of $f$ ([9]).

\section{Proposed Control Design for Connectivity Maintenance}

In the sequel, we denote with $x=\left[x_{1}, \ldots, x_{N}\right]^{T}, y=$ $\left[y_{1}, \ldots, y_{N}\right]^{T}$ the stack vectors of the $x, y$ coordinates of the agents respectively. We use the function $\operatorname{sgn}(x)=1$, if $x \geq 0$ and $\operatorname{sgn}(x)=-1$, otherwise. The function $\arctan 2(x, y)$ that is also used is the same as arc tangent of the two variables $x$ and $y$ with the distinction that the signs of both arguments are used to determine the quadrant of the result. We also use $\arctan 2(0,0)=0$. Furthermore, the notation $(a)_{i}$ for a vector $a$ denotes its $i$-th element.

In this section, we assume that each agent has limited sensing capabilities which are bounded within a cyclic area of specific radius $d$ around the agent. This cyclic area is called the sensing zone, while the parameter $d$ the sensing radius of each agent. The control design is defined in order to guarantee that if the communication graph is initially connected, then it remains connected until rendezvous is reached. In particular, we show that the control law forces the agents that are initially located within the sensing zone of an agent to remain within this area for all time. In this way, no edges are lost and the initially connected communication graph remains connected for all time. Hence, in this section, the set $N_{i}$ is defined as the set that agent $i$ can sense when it is located at its initial position, $q_{i}(0)$

$$
N_{i}=\left\{j \in \mathcal{N}, j \neq i:\left\|q_{i}(0)-q_{j}(0)\right\|<d\right\} .
$$

We consider the function $\xi:\left[0, d^{2}\right) \rightarrow[0, \infty)$ with

$$
\xi(x)= \begin{cases}\frac{a_{1}}{d^{2}-x}, & \mu<x<d^{2} \\ a_{2} x, & 0 \leq x \leq \mu\end{cases}
$$

The parameters of $\xi$ are chosen so that it is continuously differentiable for all $x \in\left[0, d^{2}\right)$. It is easily derived that this is fulfilled provided that $d^{2}=2 \mu, a_{1}=a_{2} \mu^{2}$.
An attractive potential $\delta_{i j}: \mathbb{R}^{2 N} \rightarrow \mathbb{R}$ that reflects the existence of an edge between agents $i$ and $j$ is defined as:

$$
\delta_{i j}(q) \triangleq \xi(\underbrace{\left\|q_{i}-q_{j}\right\|^{2}}_{\beta_{i j}(q)})=\xi\left(\beta_{i j}(q)\right) .
$$

In the sequel, the notation $\beta_{i j}(q)=\beta_{i j}$ is used for brevity for the squared norm of the Euclidean distance of agents $i, j$. The total attractive potential of agent $i$ is given by $\delta_{i}(q)=\sum_{j \in N_{i}} \delta_{i j}(q)=$ $\sum_{j \in N_{i}} \xi\left(\beta_{i j}\right)$. The gradient with respect to $q$ and the partial derivative of $\delta_{i j}$ with respect to $q_{i}$ are computed by $\nabla \delta_{i j}(q)=2 p_{i j} D_{i j} q$ and $\left(\partial \delta_{i j} / \partial q_{i}\right)(q)=2 p_{i j}\left(D_{i j}\right)_{i} q$ where $p_{i j} \triangleq \xi^{\prime}\left(\beta_{i j}\right)=$ $\left(d \xi\left(\beta_{i j}\right) / d \beta_{i j}\right)$ and the matrices $D_{i j},\left(D_{i j}\right)_{i}$, for $i<j$, can be shown to be given by $D_{i j}=\tilde{D}_{i j} \otimes I_{2}$, where $\left(\tilde{D}_{i j}\right)_{i i}=\left(\tilde{D}_{i j}\right)_{j j}=1$, $\left(\tilde{D}_{i j}\right)_{i j}=\left(\tilde{D}_{i j}\right)_{j i}=-1$ and $\left(\tilde{D}_{i j}\right)_{k l}=0$ for $k, l \neq i, j$, and $\left(D_{i j}\right)_{i}=\left[\begin{array}{lllll}O_{1 \times(i-1)} & 1 & O_{1 \times(j-i-1)} & -1 & O_{1 \times(N-j)}\end{array}\right] \otimes I_{2}$. The definition of $D_{i j},\left(D_{i j}\right)_{i}$ for $i>j$ is straightforward. The following symmetry property holds: $p_{i j}=p_{j i}, \forall j \in N_{i}$.

Define now $\delta(q)=\sum_{i} \delta_{i}(q)=\sum_{i} \sum_{j \in N_{i}} \delta_{i j}(q)=$ $\sum_{i} \sum_{j \in N_{i}} \xi\left(\beta_{i j}\right)$. Taking the gradient of $\delta$ with respect to $q$ we get

$$
\begin{aligned}
\nabla \delta(q) & =\sum_{i} \sum_{j \in N_{i}} \nabla \delta_{i j}(q)=2\left(\sum_{i} \sum_{j \in N_{i}} p_{i j} D_{i j}\right) q \\
& =4\left(P \otimes I_{2}\right) q
\end{aligned}
$$

where the $N \times N$ matrix $P$ is easily shown to be given by $P_{i i}=$ $\sum_{j \in N_{i}} p_{i j}, P_{i j}=-p_{i j}$ for $j \in N_{i}, i \neq j$, and $P_{i j}=0$ for $j \notin N_{i}$. In the sequel, we omit the dependence of the functions $\delta, \delta_{i}$ and $\delta_{i j}$ on $q$ for notational thrift. We also have $\left(\partial \delta_{i} / \partial q_{i}\right)=\sum_{j \in N_{i}}\left(\partial \delta_{i j} / \partial q_{i}\right)$, so that

$$
\begin{aligned}
& {\left[\left(\frac{\partial \delta_{1}}{\partial q_{1}}\right)^{T}, \ldots,\left(\frac{\partial \delta_{N}}{\partial q_{N}}\right)^{T}\right]^{T}} \\
& =\left[\left(\sum_{j \in N_{1}} \frac{\partial \delta_{1 j}}{\partial q_{1}}\right)^{T}, \ldots,\left(\sum_{j \in N_{N}} \frac{\partial \delta_{N j}}{\partial q_{N}}\right)^{T}\right]^{T} \\
& =2\left(P \otimes I_{2}\right) q .
\end{aligned}
$$

We propose the following discontinuous time-invariant feedback control law for each agent $i$ :

$$
\begin{aligned}
& u_{i}=-\operatorname{sgn}\left\{\delta_{x i} \cos \theta_{i}+\delta_{y i} \sin \theta_{i}\right\} \cdot\left(\delta_{x i}^{2}+\delta_{y i}^{2}\right)^{1 / 2} \\
& \omega_{i}=-\left(\theta_{i}-\theta_{n h_{i}}\right)
\end{aligned}
$$

where $\delta_{x i}=\left(\partial \delta_{i} / \partial x_{i}\right)=(2 P x)_{i}=2 \sum_{j \in N_{i}} p_{i j}\left(x_{i}-x_{j}\right), \delta_{y i}=$ $\left(\partial \delta_{i} / \partial y_{i}\right)=(2 P y)_{i}=2 \sum_{j \in N_{i}} p_{i j}\left(y_{i}-y_{j}\right)$ and $\theta_{n h_{i}}=$ $\arctan 2\left(\delta_{y i}, \delta_{x i}\right)$.

Before proceeding with the convergence properties of the proposed scheme, a short discussion on the choice of this control law is in order. The quadratic term in (4) is minimized whenever the agents reach a rendezvous point. In particular, we show in the sequel that the agents converge to the set $S_{o}=\left\{\delta_{x i}=\delta_{y i}=0, \forall i \in \mathcal{N}\right\}$, which is shown to correspond to a rendezvous point, provided that the initially formed communication graph is connected. For agents that have not reached the desired equilibrium $\delta_{x i}=\delta_{y i}=0$, the angular velocity control law (5) forces them to leave the undesired set inside the sign function, as will be shown in the sequel. A similar control strategy was used in [23] for stabilization of a single unicycle agent. Furthermore, the proposed control law forces pairs of agents that are initially located within 
distance less than $d$ from each other, i.e. form an edge in the communication graph, to remain within distance $d$ from each other throughout the closed loop system evolution. In other words, the control law (4), (5) is edge preserving. This fact is explicitly stated in Lemma 3.

We use the continuously differentiable positive definite function $W=\delta$ as a candidate Lyapunov function. Since the proposed controller is discontinuous we use the concept of Theorem 1 for the time derivative of $W$. Since $W$ is smooth we have $\partial W=\{\nabla W\}=\{\nabla \delta\}=\left\{\sum_{i} \nabla \delta_{i}\right\}$, so that

$$
\begin{aligned}
& \dot{\widetilde{W}}=\left\{\sum_{i}\left(\nabla \delta_{i}\right)^{T}\right\} \cdot K\left[\begin{array}{c}
u_{1} \cos \theta_{1} \\
u_{1} \sin \theta_{1} \\
\vdots \\
u_{N} \cos \theta_{N} \\
u_{N} \sin \theta_{N}
\end{array}\right] \\
& \subset 4 q^{T}\left(P \otimes I_{2}\right)\left[\begin{array}{c}
K\left[u_{1}\right] \cos \theta_{1} \\
K\left[u_{1}\right] \sin \theta_{1} \\
\vdots \\
K\left[u_{N}\right] \cos \theta_{N} \\
K\left[u_{N}\right] \sin \theta_{N}
\end{array}\right] \\
& \subset 4(P x)^{T}\left[\begin{array}{c}
K\left[u_{1}\right] \cos \theta_{1} \\
\vdots \\
K\left[u_{N}\right] \cos \theta_{N}
\end{array}\right]+4(P y)^{T}\left[\begin{array}{c}
K\left[u_{1}\right] \sin \theta_{1} \\
\vdots \\
K\left[u_{N}\right] \sin \theta_{N}
\end{array}\right] \\
& \subset \sum_{i}\left\{4 K\left[u_{i}\right]\left((P x)_{i} \cos \theta_{i}+(P y)_{i} \sin \theta_{i}\right)\right\}
\end{aligned}
$$

where we used [20, Th. 1.3] to calculate the inclusions of the Filippov set in the previous analysis. Since $K[\operatorname{sgn}(x)] x=\{|x|\}$ [20, Th. 1.7], the choice (4), (5) results in

$$
\dot{\widetilde{W}}=-\sum_{i}\left\{8\left|\delta_{x i} \cos \theta_{i}+\delta_{y i} \sin \theta_{i}\right|\left(\delta_{x i}^{2}+\delta_{y i}^{2}\right)^{1 / 2}\right\} \leq 0
$$

so that the generalized derivative of $W$ reduces to a singleton. The last equation implies that $W$ is nonincreasing across the trajectories of the closed-loop system, i.e., $W(q(t)) \leq W(q(0))$ for all $t \geq 0$.

Let $\mathcal{G}=(V, E)$ denote the initially formed communication graph under the ruling (3), according to Definition 1. Hence, an edge between agents $i, j$ exists whenever they are initially located within distance $d$ from each other, i.e., $(i, j) \in E \Leftrightarrow j \in N_{i}$ iff $\left\|q_{i}(0)-q_{j}(0)\right\|<d$. By showing that for all pairs of agents $(i, j)$ s.t. $\left\|q_{i}(0)-q_{j}(0)\right\|<d$ the proposed controller guarantees that $\left\|q_{i}(t)-q_{j}(t)\right\|<d$ for all $t>0$, the edges are guaranteed to remain invariant (i.e., agents $i, j$ remain within distance $d$ from one another) and hence the communication graph itself, remains invariant throughout the closed loop system evolution. This is explicitly shown in the next result.

Lemma 3: Let the system of multiple unicycles driven by the control law (4), (5).

The set $\mathcal{J}(q)=\left\{q \mid\left\|q_{i}-q_{j}\right\|<d, \forall(i, j) \in E\right\}$ is invariant for the trajectories of the closed-loop system.

Proof: For every initial condition $q(0) \in \mathcal{J}(q)$, we have $W(q(t)) \leq W(q(0))<\infty$ for all $t \geq 0$. Since $W \rightarrow \infty$ when $\left\|q_{i}-q_{j}\right\| \rightarrow d$ for at least one pair $(i, j) \in E$, we conclude that $q(t) \in \mathcal{J}(q)$, for all $t \geq 0$. $\diamond$

This control law guarantees that the initial set of edges, created under (3), remains invariant during the evolution of the closed loop system. Hence, no edges are lost and no new ones are created, even when an agent, not initially located in the sensing zone of another, enters in this area at some time $t>0$. The sets $N_{i}$ initially formed remain constant as the system evolves. We now state the main result of this note:

Theorem 4: Assume that the communication graph formed under the initial condition ruling (3) is connected. Then the discontinuous time-invariant feedback control strategy (4), (5) drives the agents to a common configuration in the state-space.

Proof: The level sets of $W$ are compact and invariant with respect to the relative positions of adjacent agents. Specifically, the set $\Omega_{c}=\{q: W(q) \leq c\}$ for $c>0$ is closed by the continuity of $W$. For all $(i, j) \in E$ we have $W \leq c \Rightarrow \delta_{i j} \leq c \Rightarrow\left\|q_{i}-q_{j}\right\| \leq \sqrt{\delta_{i j}^{-1}(c)}$. Connectivity of the initially formed communication graph guarantees that every pair $i, j$ of agents satisfies $\left\|q_{i}-q_{j}\right\| \leq(N-1) \delta_{\max }^{-1}$, where $\delta_{\max }^{-1} \triangleq \max _{(i, j) \in E} \sqrt{\delta_{i j}^{-1}(c)}$. Therefore, the set $\Omega_{c}$ is also bounded with respect to the relative positions of agents in the group. Hence for all initial conditions satisfying $\left\|q_{i}(0)-q_{j}(0)\right\|<d, \forall(i, j) \in E$ we can apply (the nonsmooth version of) LaSalle's invariance principle (Theorem 2). By Theorem 2, the trajectories of the system converge to the largest invariant set contained in the set $S=\left\{\left(\delta_{x i}=\delta_{y i}=\right.\right.$ $\left.0) \vee\left(\delta_{x i} \cos \theta_{i}+\delta_{y i} \sin \theta_{i}=0\right), \forall i \in N\right\}$. However, for each $i \in \mathcal{N}$, we have $\left|\omega_{i}\right|=\pi / 2$ whenever $\delta_{x i} \cos \theta_{i}+\delta_{y i} \sin \theta_{i}=0$, due to the proposed angular velocity control law. In particular, this choice of angular velocity renders the surface $\delta_{x i} \cos \theta_{i}+\delta_{y i} \sin \theta_{i}=0$ noninvariant for agent $i$, whenever $i$ is not located at the desired equilibrium, namely when $\delta_{x i}=\delta_{y i}=0$. Hence, the largest invariant set $S_{o}$ contained in $S$ is $S \supset S_{o}=\left\{\delta_{x i}=\delta_{y i}=0, \forall i \in \mathcal{N}\right\}$. In addition $\left(\delta_{x i}=\delta_{y i}=0\right) \forall i$ guarantees that the agents converge to a common configuration. This is easily derived by the fact that $\left(\delta_{x i}=\delta_{y i}=\right.$ $0) \forall i \Rightarrow\left(P \otimes I_{2}\right) q=0 \Rightarrow P x=P y=0$, where $x, y$ the stack vectors of $q$ in the $x, y$ directions. Within $S_{o}$, the closed-loop equations for the orientations have the form $\dot{\theta}_{i}=-\theta_{i}$ for all $i$, and hence the orientations of all agents tend to zero. The symmetric matrix $P$ has zero row sums and non-positive off-diagonal elements. Using the same arguments and terminology as in [17], the matrix $P$ is a Metzler matrix. As mentioned in Section III-A, the eigenvalues of $P$ are nonnegative and zero is the smallest eigenvalue. Following [17], we deduce that since the initially formed communication graph is connected, zero is a simple eigenvalue of $P$ with trivial corresponding eigenvector the vector of ones, $\overrightarrow{\mathbf{1}}$. Hence, equations $P x=P y=0$ guarantee that both $x, y$ are eigenvectors of $P$ belonging to $\operatorname{span}\{\overrightarrow{\mathbf{1}}\}$. Hence, all $q_{i}$ tend to the same value, implying that all agents converge to a common configuration at steady state. $\diamond$

\section{Static and Switching Topology}

In this section, we show that the weaker cases of static and switching interconnection topologies can be treated as corollaries of the framework presented previously. The difference lies in the definition of the sets $N_{i}$ as well as the choice of the inter-agent potentials $\delta_{i j}$.

1) Static Topology: In the static topology case, we assume that each agent $i$ communicates with a subset $N_{i}$ of the rest of the team throughout the closed loop system evolution. Hence, the set $N_{i}$ is static and independent of the agents' relative positions. The interagent potentials are now simply given by $\delta_{i j}(q)=\beta_{i j}$ for $(i, j) \in E$ and $\delta_{i j}(q)=$ 0 , otherwise. We have $\delta_{i}(q)=\sum_{j \in N_{i}} \delta_{i j}(q)=\sum_{j \in N_{i}} \beta_{i j}$ and the function $\delta$ is given by $\delta(q)=\sum_{i} \delta_{i}(q)=\sum_{i} \sum_{j \in N_{i}} \delta_{i j}(q)=$ $\sum_{i} \sum_{j \in N_{i}} \beta_{i j}$. The gradient of $\delta$ in this case is given by

$$
\nabla \delta(q)=\sum_{i} \sum_{j \in N_{i}} \nabla \delta_{i j}(q)=\sum_{i} \sum_{j \in N_{i}} \nabla \beta_{i j}=4\left(\mathcal{L} \otimes I_{2}\right) q
$$



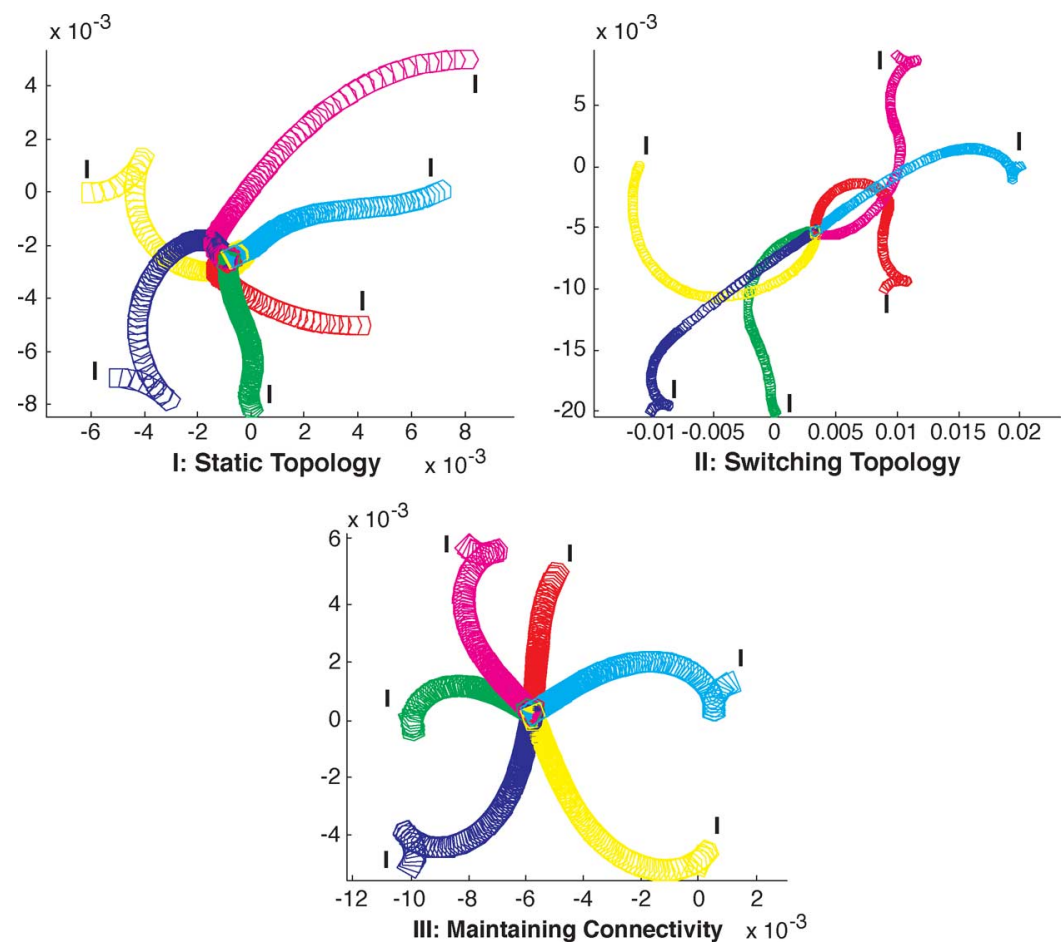

Fig. 1. Simulations of three scenarios based on Corollaries 5 and 6 and Theorem 4 respectively.

where $\mathcal{L}$ is the Laplacian matrix of the communication graph. We also have $\left(\partial \delta_{i} / \partial q_{i}\right)=\sum_{j \in N_{i}}\left(\partial \delta_{i j} / \partial q_{i}\right)$, so that we can again calculate

$$
\begin{aligned}
& {\left[\left(\frac{\partial \delta_{1}}{\partial q_{1}}\right)^{T}, \ldots,\left(\frac{\partial \delta_{N}}{\partial q_{N}}\right)^{T}\right]^{T}} \\
& \quad=\left[\left(\sum_{j \in N_{1}} \frac{\partial \delta_{1 j}}{\partial q_{1}}\right)^{T}, \ldots,\left(\sum_{j \in N_{N}} \frac{\partial \delta_{N j}}{\partial q_{N}}\right)^{T}\right]^{T} \\
& =2\left(\mathcal{L} \otimes I_{2}\right) q .
\end{aligned}
$$

An immediate corollary of Theorem 4 for the static case is now stated as follows.

Corollary 5: Assume that the communication graph is connected and let $\mathcal{L}$ denote its Laplacian matrix. Then the discontinuous time-invariant feedback control strategy (4), (5), where the terms $\delta_{x i}, \delta_{y i}$ are now given by $\delta_{x i}=\left(\partial \delta_{i} / \partial x_{i}\right)=(\mathcal{L} x)_{i}=\sum_{j \in N_{i}}\left(x_{i}-x_{j}\right), \delta_{y i}=$ $\left(\partial \delta_{i} / \partial y_{i}\right)=(\mathcal{L} y)_{i}=\sum_{j \in N_{i}}\left(y_{i}-y_{j}\right)$, and the "nonholonomic angle" $\theta_{n h_{i}}=\arctan 2\left(\delta_{y i}, \delta_{x i}\right)^{i}$, drives the agents to a common configuration in the state-space.

Proof: Using $W=\delta$ as a candidate Lyapunov function, the exact same procedure as in the proof of Theorem 4 shows that the generalized time derivative of $W$ satisfies (6). Since $W$ is quadratic in the agents' relative positions, its level sets are compact and invariant for the trajectories of the closed-loop system. Specifically, we have $W \leq c \Rightarrow\left\|q_{i}-q_{j}\right\| \leq \sqrt{2 c}, \forall(i, j) \in E$. Connectivity of the communication graph ensures that the maximum length of a path connecting two vertices of the graph is at most $N-1$. Hence $\left\|q_{i}-q_{j}\right\| \leq$ $\sqrt{2 c}(N-1), \forall i, j \in \mathcal{N}$. Using now Theorem 2 , the same arguments as in the proof of Theorem 4 guarantee that the agents converge to the set $S_{o}=\left\{\delta_{x i}=\delta_{y i}=0, \forall i \in N\right\}$ with zero orientation. Furthermore, $\left(\delta_{x i}=\delta_{y i}=0\right), \forall i \Rightarrow\left(\mathcal{L} \otimes I_{2}\right) q=0 \Rightarrow \mathcal{L} x=\mathcal{L} y=0$ where $x, y$ the stack vectors of $q$ in the $x, y$ directions. The fact that the communication graph is connected implies that $\mathcal{L}$ has a simple zero eigenvalue with corresponding eigenvector the vector of ones, $\overrightarrow{\mathbf{1}}$. This guarantees that both $x, y$ are eigenvectors of $\mathcal{L}$ belonging to $\operatorname{span}\{\overrightarrow{\mathbf{1}}\}$. Hence for all $i \in \mathcal{N}$, all $q_{i}$ have a common value, implying that all agents converge to a common configuration at steady state. $\diamond$

2) The Case of Switching Topology: In this section, the set $N_{i}$ is time varying and dependent on the relative positions of the agents. Specifically, we assume that inter-agent communication is created/lost each time an agent enters/leaves the sensing zone radius $d$ around another agent. Hence for agent $i$, the set $N_{i}$ is defined as

$$
N_{i}=\left\{j:\left\|q_{i}-q_{j}\right\| \leq d\right\} .
$$

Hence, an edge is created in the communication graph each time an agent enters the sensing zone of another agent. We use the function $\sigma:[0, \infty) \rightarrow \mathbb{R}$ which is defined as

$$
\sigma(x)= \begin{cases}\frac{1}{2} x, & 0 \leq x \leq c^{2} \\ a_{5} x^{2}+a_{4} x+a_{3}, & c^{2} \leq x \leq d^{2} \\ h, & d^{2} \leq x\end{cases}
$$

We require that this function is continuously differentiable in its whole domain. This is fulfilled by choosing: $a_{5}=\left(1 / 4\left(c^{2}-d^{2}\right)\right), a_{4}=$ $\left(d^{2} / 2\left(d^{2}-c^{2}\right)\right), a_{3}=\left(c^{4} / 4\left(c^{2}-d^{2}\right)\right),\left(h=\left(d^{2}+c^{2}\right) / 4\right)$.

In order to cope with the limited sensing capabilities of each agent, the function $\delta_{i j}: \mathbb{R}^{2 N} \rightarrow \mathbb{R}$ is redefined as $\delta_{i j}(q) \triangleq \sigma\left(\beta_{i j}\right)$. The gradient and the partial derivative of $\delta_{i j}$ are computed by $\nabla \delta_{i j}=$ $2 \rho_{i j} D_{i j} q$ and $\left(\partial \delta_{i j} / \partial q_{i}\right)=2 \rho_{i j}\left(D_{i j}\right)_{i} q$ where $\rho_{i j} \triangleq \sigma^{\prime}\left(\beta_{i j}\right)=$ $\left(d \sigma\left(\beta_{i j}\right) / d \beta_{i j}\right)$ and the matrices $D_{i j},\left(D_{i j}\right)_{i}$ were defined previously. This choice of $\sigma$ guarantees that $\rho_{i j}>0$ for $0<\beta_{i j}<d^{2}$ and $\rho_{i j}=0$ for $\beta_{i j} \geq d^{2}$. In this section, the function $\delta_{i}$ is defined in a different manner than the previous two cases. In particular, we have $\delta_{i}(q)=\sum_{j \neq i} \delta_{i j}(q)=\sum_{j \neq i} \sigma\left(\beta_{i j}\right)$. However, using the fact that $\rho_{i j}=0$ for $\beta_{i j}>d^{2}$, the following equation is straightforward:

$$
\frac{\partial \delta_{i}}{\partial q_{i}}=\sum_{j \neq i} \frac{\partial \delta_{i j}}{\partial q_{i}}=\sum_{j \in N_{i}} \frac{\partial \delta_{i j}}{\partial q_{i}}
$$


so that each agent has to take into account only agents belonging to $N_{i}$ at each time instant in order to implement the control law (4), (5) with $\delta_{x i}=\left(\partial \delta_{i} / \partial x_{i}\right), \delta_{y i}=\left(\partial \delta_{i} / \partial y_{i}\right)$. Using now $\delta(q)=\sum_{i} \delta_{i}(q)$ and taking its gradient, we can compute

$$
\begin{aligned}
\nabla \delta(q) & =\sum_{i} \sum_{j \in N_{i}} \nabla \delta_{i j}(q)=2\left(\sum_{i} \sum_{j \in N_{i}} \rho_{i j} D_{i j}\right) q \\
& =2\left(\sum_{i} \sum_{j \neq i} \rho_{i j} D_{i j}\right) q=4\left(R \otimes I_{2}\right) q
\end{aligned}
$$

where we have used again the fact that $\rho_{i j}=0$ for $\beta_{i j}>d^{2}$ and the relation $\rho_{i j}=\rho_{j i}$. The elements of the $N \times N$ matrix $R$ are given by $R_{i i}=\sum_{j \neq i} \rho_{i j}$ and $R_{i j}=-\rho_{i j}$, for $i \neq j$. Also

$$
\begin{aligned}
& {\left[\left(\frac{\partial \delta_{1}}{\partial q_{1}}\right)^{T}, \ldots,\left(\frac{\partial \delta_{N}}{\partial q_{N}}\right)^{T}\right]^{T}} \\
& =\left[\left(\sum_{j \neq 1} \frac{\partial \delta_{1 j}}{\partial q_{1}}\right)^{T}, \ldots,\left(\sum_{j \neq N} \frac{\partial \delta_{N j}}{\partial q_{N}}\right)^{T}\right]^{T} \\
& =\left[\left(\sum_{j \in N_{1}} \frac{\partial \delta_{1 j}}{\partial q_{1}}\right)^{T}, \ldots,\left(\sum_{j \in N_{N}} \frac{\partial \delta_{N j}}{\partial q_{N}}\right)^{T}\right]^{T} \\
& =2\left(R \otimes I_{2}\right) q .
\end{aligned}
$$

The following corollary holds for the switching topology case.

Corollary 6: Assume that the communication graph remains connected for every topology induced by the switching communication law (7). Then the control law (4), (5), where the terms $\delta_{x i}, \delta_{y i}$ are now given by $\delta_{x i}=\left(\partial \delta_{i} / \partial x_{i}\right)=(2 R x)_{i}=2 \sum_{j \in N_{i}} \rho_{i j}\left(x_{i}-x_{j}\right)$, $\delta_{y i}=\left(\partial \delta_{i} / \partial y_{i}\right)=(2 R y)_{i}=2 \sum_{j \in N_{i}} \rho_{i j}\left(y_{i}-y_{j}\right)$ and $\theta_{n h_{i}}=$ $\arctan 2\left(\delta_{y i}, \delta_{x i}\right)$, drives the agents to a common configuration in the state-space.

Proof: For $W=\delta$, the same arguments as in the proof of Theorem 4 show that the generalized time derivative of $W$ satisfies again (6). The assumption that the communication graph remains connected guarantees that the set $\Omega=\left\{q:\left\|q_{i}-q_{j}\right\| \leq(N-1) d, \forall i, j \in \mathcal{N}\right\}$ is compact and invariant for the closed loop system with respect to the relative positions of all agents belonging to $\mathcal{N}$. The same procedure as in the proof of Theorem 4 guarantees that the agents converge to the set $S_{o}=\left\{\delta_{x i}=\delta_{y i}=0, \forall i \in N\right\}$ with zero orientation. In addition, $\left(\delta_{x i}=\delta_{y i}=0\right), \forall i \Rightarrow\left(R \otimes I_{2}\right) q=0 \Rightarrow R x=R y=0$ where $x$, $y$ the stack vectors of $q$ in the $x, y$ directions. The symmetric matrix $R$ has zero row sums and non-positive off-diagonal elements and hence is a Metzler matrix. Using the same arguments as in the end of Theorem 4 , we deduce that since the communication graph remains connected, zero is a simple eigenvalue of $R$ with trivial corresponding eigenvector the vector of ones, $\overrightarrow{\mathbf{1}}$. Hence equations $R x=R y=0$ guarantee that both $x, y$ are eigenvectors of $R$ belonging to $\operatorname{span}\{\overrightarrow{\mathbf{1}}\}$. Hence all $q_{i}$ tend to the same value, implying that all agents converge to a common configuration at steady state. $\diamond$

\section{E. Discussion}

It should be noted that the cases of static and switching interconnection links of the previous section are weaker cases than the case of connectivity maintenance that was dealt with in Section III-C. Specifically, connectivity of the communication graph is an assumption in Corollaries 5 and 6 , while it is a guaranteed invariant property of the closed loop system in Theorem 4, by virtue of Lemma 3. In essence, Theorem 4 is a stronger and more applicable result. Moreover, as witnessed previously, all three cases can be viewed as special cases of the control framework (4), (5) with a particular definition of the function $\delta_{i j}$ and the communication set $N_{i}$. We should also note that the proposed control law (4), (5) is purely decentralized, since each agent $i$ requires information only of the states of agents within $N_{i}$ at each time instant.

\section{Simulations}

The results of the previous sections are demonstrated through the three simulation scenarios presented in Fig. 1. In each screenshot, the initial position of each agent is denoted by the letter "I." The first screenshot I presents a scenario of six unicycles navigating under the static communication control scheme. The communication sets in this simulation have been chosen so that the resulting communication graph is connected. Agents are eventually driven to a rendezvous point, in agreement with Corollary 5. In the second scenario (screenshot II), the agents navigate under the switching communication strategy. The communication graph remains connected throughout the evolution of the closed loop system, and hence rendezvous is reached, as implied by Corollary 6 . This theorem cannot guarantee that the graph remains connected-this is only an assumption in Corollary 6 . The result of Theorem 4 guarantees that if the communication graph is initially connected, then the control laws (4), (5) preserve connectivity. This is demonstrated in the third scenario of screenshot III. Connectivity of the initially formed communication graph guarantees connectivity preserving and rendezvous convergence.

\section{CONCLUSION}

In this note, a decentralized feedback control strategy that drives a system of multiple nonholonomic unicycles to a rendezvous point in terms of both position and orientation was introduced. The proposed nonholonomic control law is discontinuous and time-invariant and using tools from nonsmooth Lyapunov theory and graph theory the stability of the overall system is examined. Similarly to the linear case, the convergence of the multiagent system relies on the connectivity of the communication graph that represents the interagent communication topology. The control law was first defined in order to guarantee connectivity maintenance for an initially connected communication graph. Moreover, the cases of static and dynamic communication topologies were treated as corollaries of the proposed framework.

\section{REFERENCES}

[1] H. Ando, Y. Oasa, I. Suzuki, and Yamashita, "Distributed memoryless point convergence algorithm for mobile robots with limited visibility," IEEE Trans. Robot. Automat., vol. 15, no. 5, pp. 818-828, Oct. 1999.

[2] B. Bollobás, Modern Graph Theory, ser. Springer Graduate Texts in Mathematics 184. New York: Springer-Verlag, 1998.

[3] R. W. Brockett, "Control theory and singular riemannian geometry," in New Directions in Applied Mathematics. New York: Springer-Verlag, 1981, pp. 11-27.

[4] F. Ceragioli, "Discontinuous Ordinary Differential Equations and Stabilization," Ph.D. thesis, Dept. Math., Univ. di Firenze, Firenze, Italy, 1999.

[5] F. Clarke, Optimization and Nonsmooth Analysis. Reading, MA: Addison-Wesley, 1983.

[6] J. Cortes, S. Martinez, and F. Bullo, "Robust rendezvous for mobile autonomous agents via proximity graphs in arbitrary dimensions," IEEE Trans. Autom. Control, vol. 51, no. 8, pp. 1289-1298, Aug. 2006.

[7] D. V. Dimarogonas and K. J. Kyriakopoulos, "On the state agreement problem for multiple unicycles," in Proc. 2006 Amer. Control Conf., pp. 2016-2021.

[8] D. V. Dimarogonas and K. J. Kyriakopoulos, "On the state agreement problem for multiple unicycles with varying communication links," in Proc. 45th IEEE Conf. Decision Control, 2006, pp. 4283-4288.

[9] A. Filippov, Differential Equations With Discontinuous Right-Hand Sides. Norwell, MA: Kluwer, 1988.

[10] R. A. Horn and C. R. Johnson, Matrix Analysis. Cambridge, U.K.: Cambridge Univ. Press, 1996. 
[11] A. Jadbábaie, J. Lin, and A. S. Morse, "Coordination of groups of mobile autonomous agents using nearest neighbor rules," IEEE Trans. Autom. Control, vol. 48, no. 6, pp. 988-1001, Jun. 2003.

[12] M. Ji and M. Egerstedt, "Connectedness preserving distibuted coordination control over dynamic graphs," in Proc. Amer. Control Conf., 2005, pp. 93-98.

[13] B. Kim and P. Tsiotras, "Controllers for unicycle-type wheeled robots: Theoretical results and experimental validation," IEEE Trans. Robot. Automat., vol. 18, no. 3, pp. 294-307, Jun. 2002.

[14] G. Lafferriere, A. Williams, J. Caughman, and J. J. P. Veerman, "Decentralized control of vehicle formations," Syst. Control Lett., vol. 54, no. 9, pp. 899-910, 2005.

[15] Z. Lin, B. Francis, and M. Maggiore, "Necessary and sufficient graphical conditions for formation control of unicycles," IEEE Trans. Autom. Control, vol. 50, no. 1, pp. 121-127, Jan. 2005.

[16] D. G. Luenberger, Introduction to Dynamic Systems: Theory, Models and Applications. New York: Wiley, 1979.

[17] L. Moreau, "Stability of continuous-time distributed consensus algorithms," in Proc. 43rd IEEE Conf. Decision Control, 2004, pp. 3998-4003.

[18] A. Muhammad and M. Egerstedt, "Connectivity graphs as models of local interactions," in Proc. 43rd IEEE Conf. Decision Control, 2004, pp. 124-129.

[19] R. Olfati-Saber and R. M. Murray, "Consensus problems in networks of agents with switching topology and time-delays," IEEE Trans. Autom. Control, vol. 49, no. 9, pp. 1520-1533, Sep. 2004.

[20] B. Paden and S. S. Sastry, "A calculus for computing Filippov's differential inclusion with application to the variable structure control of robot manipulators," IEEE Trans. Circuits Syst., vol. 34, no. 1, pp. 73-82, Jan. 1987.

[21] W. Ren, R. W. Beard, and E. M. Atkins, "A survey of consensus problems in multi-agent coordination," in Proc. Amer. Control Conf., 2005, pp. $1859-1864$.

[22] D. Shevitz and B. Paden, "Lyapunov stability theory of nonsmooth systems," IEEE Trans. Autom. Control, vol. 49, no. 9, pp. 1910-1914, Sep. 1994.

[23] H. Tanner and K. J. Kyriakopoulos, "Backstepping for nonsmooth systems," Automatica, vol. 39, pp. 1259-1265, 2003.

[24] H. Yamaguchi and J. W. Burdick, "Asymptotic stabilization of multiple nonholonomic mobile robots forming group formations," in Proc. IEEE Int. Conf. Robot. Automat., 1998, pp. 3573-3580.

\section{Block Decoupling by Precompensation Revisited}

Michel Malabre and Jorge A. Torres-Muñoz

\begin{abstract}
The block decoupling problem by admissible dynamic precompensation for LTI systems is considered. Admissibility refers to the preservation of the class of controlled output trajectories, i.e. functional output controllability is concerned, which is more demanding than just pointwise output controllability. This problem has been solved by Hautus and Heyman, within a transfer function matrix approach. Different new equivalent solvability conditions in terms of controllability subspaces, transfer function matrices or matrix pencils are given. One of these conditions (expressed in the input space) is at the origin of new necessary and sufficient conditions for block decoupling by general precompensation (possibly non admissible and nonsquare), in the wider sense of Basile and Marro.
\end{abstract}

Index Terms-Block decoupling, controllability subspaces, linear timeinvariant (LTI) systems, precompensation, transfer function matrices.

\section{INTRODUCTION}

Input-output decoupling problem has attracted the interest of the control community from the very beginning of the development of the linear systems theory. Actually, there is a huge body of theoretical results within the so-called geometric approach as well as in the transfer function matrix approach. In this sense, recommended accounts can be found in [4], [13].

Block decoupling amounts to finding a control law in such a way that on the compensated system subsets of inputs drive specified blocks of outputs without interacting with other output blocks. In order to avoid trivial controllers such as the zero one, driving requirements for the outputs must be imposed, such as pointwise or functional output controllability preservation between the system and the compensated system.

A dynamic precompensation scheme is considered here, which includes dynamic (static) state (measurement) feedback as particular cases.

An elegant solution to block decoupling has been proposed by Basile and Marro in [7] within the geometric approach that relies on the properties of some specific controllability subspaces of the system. Their solution is general in the sense that the requirement on the controller is the preservation of the output pointwise controllability. Later, Hautus and Heymann [1] gave a solution to the block decoupling problem by admissible precompensation that requires the preservation of the output functional controllability properties. Their solution is based on the notion of independent rational spaces generated by the row-blocks of the transfer function matrix.

The main aim of the present contribution is to bridge some gap between these two different approaches. We first derive some equivalent characterizations in the transfer function, in the geometric as well as in

Manuscript received March 22, 2005; revised May 18, 2006 and January 1, 2007. Recommended by Associate Editor A. Garulli. This work was supported by CNRS (France)-CONACYT (Mexico), within binational laboratory LAFMAA.

M. Malabre is with IRCCyN, Institut de Recherche en Communications et Cybern étique de Nantes, UMR CNRS 6597, B.P. 92 101, 44321 Nantes Cedex 03, France (e-mail: michel.malabre@irccyn.ec-nantes.fr).

J. A. Torres-Muñoz is with the Department of Automatic Control, CINVESTAV-IPN, 07300 Mexico City, Mexico (e-mail: jtorres@ ctrl.cinvestav.mx).

Digital Object Identifier 10.1109/TAC.2007.895893 